\title{
Socio-demographic and Clinical Profile of Bangladeshi COVID 19 Patients with their Clinical Outcome in National Institute of Cardiovascular Diseases, Dhaka
}

\author{
Ferdush Jahan ${ }^{1}$, Md. Shahed Kamal Bhuya ${ }^{2}$, Muhammed Shahed Anwar Bhuya ${ }^{3}$, Jamal Uddin \\ Gaji ${ }^{1}$, Rumana Nushrat ${ }^{4}$, Zulfikar $\mathrm{Ali}^{5}$, Nasrin Sultana ${ }^{6}$ \\ ${ }^{1}$ Dept. of Microbiology, NICVD, Dhaka, ${ }^{2}$ Dept. of Vascular Surgery, NICVD, Dhaka, ${ }^{3}$ Dept. \\ Orthopaedic Surgery, Cumilla Medical College and Hospital, Cumilla, ${ }^{4}$ Dept. of Paediatric Cardiology, \\ NICVD, Dhaka, ${ }^{5}$ Dept. of Cardiology, NICVD, Dhaka, ${ }^{6}$ Dept. of Radiology and Imaging, Probin \\ Hospital (BAAGM), Dhaka
}

Key word: COVID 19, Bangladesh.

\begin{abstract}
:
Background: The novel corona virus (COVID 19) pandemic is a major global health threat of the twenty-first century. Clinical presentation, rapid identification of causes and isolation are vital for containments of rapidly spreading disease. The objective of the study was to report early findings on demographic profile, clinical presentation of the confirmed COVID 19 patients with their clinical outcome.

Methods: This observational study was conducted in Microbiology Department of National Institute of Cardiovascular Diseases (NICVD) for the period of October 01, 2020 to November 30, 2020. Total 300 positive COVID 19 patients were included and interviewed. Informed written consent was ensured before participation. After collection, Data were analyzed to show the characteristics of COVID 19 cases and their clinical outcome after treatment.

Results: Among the 300 cases 228 (76\%) patients were male and $72(24 \%)$ patients were female. Average age of the patients was 39 years. The most commonly observed symptoms were fever (70\%), followed by cough (55\%), breathlessness (42\%), dysgeusia (38\%), anosmia (25\%). Respiratory symptom was the dominant feature of clinical presentation. The most prevalent affected age groups were 114 (38\%) patients in 41-50 years age, 102 (34\%) patients in 31- 40 years age. Among the total cases 255 (85\%) patients were Urban residents and 234 (78\%) had contact history. Among 300 patients 102 (34\%) patients had co-morbidities and presence of co-morbidities $(p<0.01)$ were significantly associated with mortality. The death rate was $2 \%$.

Conclusion: Typical presentations of COVID-19 were fever, cough, breathlessness, dysgeusia and anosmia. Requirement of ICU was $6 \%$ and overall mortality was $2 \%$ which was associated with comorbidities.
\end{abstract}

(Cardiovasc j 2021; 14(1): 50-54)

\section{Introduction:}

The novel corona virus pandemic is a major global health threat of the twenty-first century and was first detected on 31 December 2019 in Wuhan, China. ${ }^{1,2}$ On $31^{\text {st }}$ December 2019, 27 cases of pneumonia of unknown etiology were identified in Wuhan city, Hubei province in China. ${ }^{3}$ The causative agent was identified from throat swab samples conducted by the Chinese Centre for Disease Control and prevention (CCDC) on $7^{\text {th }}$ January 2020 and was subsequently named Severe Acute Respiratory Syndrome Corona virus 2 (SARS

Address of Correspondence: Dr. Ferdush Jahan, Department of Microbiology, National Institute of Cardiovascular Diseases Dhaka, Bangladesh. Email- jahandrferdush@gmail.com

- 2020 authors; licensed and published by International Society of Cardiovascular Ultrasound, Bangladesh Chapter and Bangladesh Society of Geriatric Cardiology. This is an Open Access article distributed under the terms of the CC BY NC 4.0 (https://creativecommons.org/licenses/by-nc/4.0) 
CoV-2). The disease was named COVID 19 by the World Health Organization (WHO). ${ }^{4}$ COVID 19 is mainly transmitted through respiratory droplets and contact. ${ }^{5}$ At present patients with COVID 19 are the main source of infection. ${ }^{6}$

In Bangladesh, from the first case identified on $8^{\text {th }}$ March, 2020. 2,90,360 individuals have tested positive for COVID 19; 172615 cured and 3,861 died with infection fatality rate of $1.32 \%$, till August 22, 2020. ${ }^{7}$ Despite extensive efforts to prevent onward rapid spread , 213 countries and territories with $23,121,366$ confirmed cases and 803,223 deaths till 22 August 2020 has been reported worldwide. ${ }^{8}$ Regional clinical features are important to understand the short outcome of patient and their association with mortality, as asymptomatic and mild symptomatic cases are rising exponentially. ${ }^{9}$

\section{Study methods:}

Study design and participants

This study was conducted in National Institute of Cardiovascular Diseases (NICVD), Dhaka, a tertiary level hospital, Bangladesh from October 01, 2020 to November 30, 2020. We selected 300 patients from Microbiology department of this hospital. All patients were confirmed real-time polymerase chain reaction and were diagnosed as having COVID 19 according to WHO interim guidance. Under aged $(<20)$ and pregnant women were excluded from the study. Diagnosis of COVID 19 was based on the results of real time RT-PCR using nasopharyngeal swab samples. ${ }^{10}$

\section{Data collection methods:}

Data were collected either from patients or their attendants/ relatives through direct interview or reached over phone by a semi-structured questionnaire. In all cases, informed written consent was ensured before participation. A preformed questionnaire was used for data collection. The clinical parameters included age, sex, duration of illness, onset to hospital admission, co-morbidities (systemic hypertension, diabetes mellitus, heart disease and chronic obstructive pulmonary disease etc.), symptoms, clinical signs and outcome (mortality) collected through the questionnaire and were evaluated by trained physicians. Outcome was designated as alive or dead within the hospital admission. All collected data were recorded in structured case record form and later on accumulated and compiled.

\section{Case definitions:}

Confirmed COVID 19 case was detected in according to the case definition of the National guidelines of clinical Management of coronavirus disease 2019, Bangladesh. ${ }^{11}$

\section{Statistical analysis:}

Analysis was performed with statistical analysis SPSS 20 (SPSS Inc. Chicago Illinois, USA).

\section{Results:}

Total 300 COVID 19 patients were enrolled. Among them $228(76 \%)$ were male and $72(24 \%)$ patients were female. Most patients were from 41-50 years which was 114 (38\%) then from 31-40 years age group 102 (34\%), from 21-30 years age group 69 (23\%), from 51-60 years age group 9 (3\%) and 6 (2\%) patients above 60 years. [ Table I].

Table-I

Age and sex distribution of patients with COVID $19(n=300)$.

\begin{tabular}{lcc}
\hline Age (in years) & Frequency & Percent \\
\hline 21-30 years & 69 & 23 \\
31-40 years & 102 & 34 \\
41-50 years & 114 & 38 \\
51-60 years & 9 & 3 \\
$>$ 60 years & 6 & 2 \\
Male & 228 & 76 \\
Female & 72 & 24 \\
\hline
\end{tabular}

Average age of the patients was 39 years. Fever was the dominant clinical presenting feature. 216 (72\%) patients presented with fever, followed in decreasing order by cough 165 (55\%), breathlessness 126 (42\%), dysgeusia 114 (38\%), anosmia $75(25 \%)$, parosmia $66(22 \%)$, chest pain 60 (20\%), Diarrhoea 57 (19\%), and Sore throat 45 $(15 \%)$. The duration of illness was 1 to 3 weeks in $60 \%$ cases, less than 1 week in $35 \%$ cases and $>3$ weeks in $5 \%$ cases. [Table II].

Among 300 patients, 255 (85\%) patients were Urban residents. Majority 84 (28\%) of the patients had graduation and 234 (78\%) patients had contact history [Table- III] 
Table-II

Clinical characteristics of patients $(n=300)$.

\begin{tabular}{lcc}
\hline Duration of illness & Frequency & Percent \\
\hline$<1$ week & 105 & 35 \\
1 to 3 weeks & 180 & 60 \\
$>3$ weeks & 15 & 5 \\
Fever & 216 & 72 \\
Cough & 165 & 55 \\
Breathlessness & 126 & 42 \\
Dysgeusia & 114 & 38 \\
Anosmia & 75 & 25 \\
Parosmia & 66 & 22 \\
Chest pain & 60 & 20 \\
Diarrhoea & 57 & 19 \\
Sore throat & 45 & 15 \\
\hline
\end{tabular}

Table-III

Sociodemographic profile of COVID 19 patients $(n=300)$.

\begin{tabular}{lc}
\hline Variables & Findings \\
\hline Residence & $255(85 \%)$ \\
Urban & $45(15 \%)$ \\
Rural & \\
Education & $33(11 \%)$ \\
No formal education & $60(20 \%)$ \\
Completed primary education & $69(23 \%)$ \\
SSC & $54(18 \%)$ \\
HSC & $84(28 \%)$ \\
Graduation and above & $252(84 \%)$ \\
Employed & $48(16 \%)$ \\
Unemployed & \\
\hline
\end{tabular}

Total 102 (34\%) patients had one or more than one co-morbidity where Hypertension (20\%), diabetes mellitus (18\%), heart disease (6\%), renal disease (9\%), COPD (7\%) were frequent. Regarding co-morbidity status hypertension, heart disease, renal disease was significantly $(p<0.01)$ associated with death. [Table-IV]

Among 300 patients, 294 (98\%) patients were alive and $6(2 \%)$ patients died. ICU support was needed $12(4 \%)$ of the patients. [Table-V].
Table-IV

Comorbid condition of the study patients $(n=300)$.

\begin{tabular}{lcc}
\hline Comorbidities & Frequency & Percent \\
\hline Hypertension & 60 & 20 \\
Diabetes & 51 & 17 \\
Heart disease & 18 & 06 \\
Renal disease & 24 & 08 \\
COPD & 15 & 05 \\
Liver disease & 12 & 04 \\
Obesity & 18 & 06 \\
Hypothyroidism & 09 & 03 \\
Cancer & 06 & 02 \\
Total & 120 & 40 \\
\hline
\end{tabular}

Table-V

Factors associated with outcome of COVID 19 patients $(n=300)$.

\begin{tabular}{lccr}
\hline & Alive & Death & p-value \\
\hline Comorbidity present & 97 & 05 & $<0.01$ \\
Comorbidity absent & 197 & 01 & \\
ICU needed & 08 & 04 & $<0.01$ \\
ICU not needed & 286 & 02 & \\
\hline
\end{tabular}

p- value was determined by chi-square test

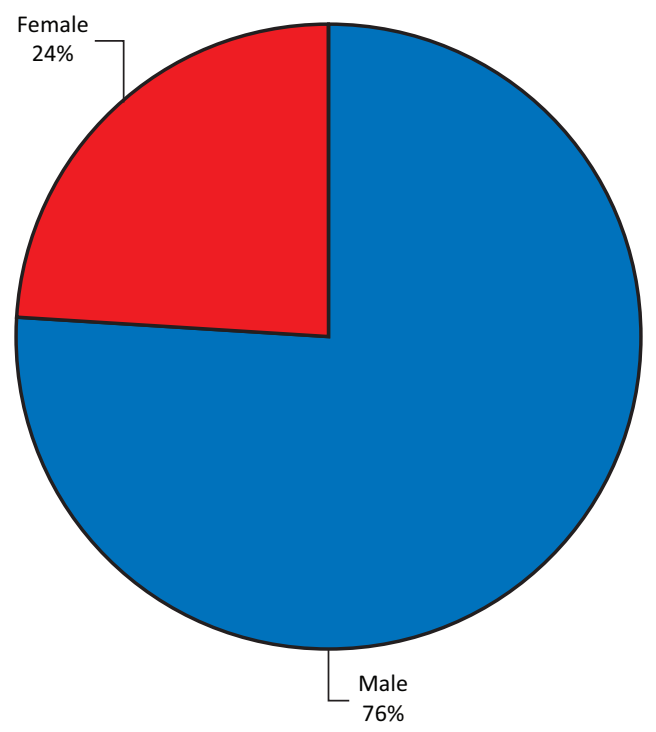

Fig.-1: Show sex distribution of cases $(n=300)$ Male were $228(76 \%)$, Female were 72(24\%). 


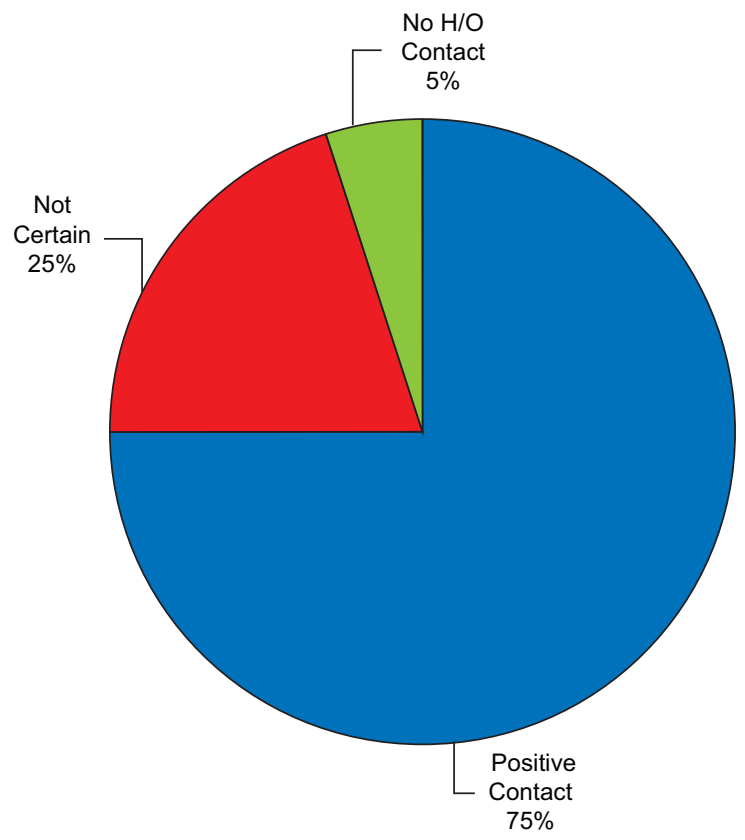

Fig.-2: Show H/O contact with COVID 19 patients.

\section{Discussion:}

In our study 228 (76\%) male patients were infected by COVID 19. Male patients were more affected than female. This study was consistent with the results of a study conducted in India performed by Gupta et al. ${ }^{12}$ Another study of Murad et al. conducted in Bangladesh reported similar findings where $77 \%$ patients were men. ${ }^{13}$ Average age of the patients were 39 years. Age group distribution showed that younger age groups were more affected than older age groups. Majority (38\%) of the patients were aged between $41-50$ years. This is consistent with the data from the Institute of Epidemiology, Disease Control and Research (IEDCR), Bangladesh on COVID 19. ${ }^{14}$

It is important to note that $78 \%$ patients had positive contact history in our study highlighting the importance of preventive and containment process of pandemic including distancing, hand washing and proper use of mask etc.

In our study, Majority had fever (72\%), followed in decreasing order by cough (55\%), breathlessness (42\%), dysgeusia (38\%), anosmia (25\%), parosmia $(22 \%)$, chest pain $(20 \%)$, diarrhoea $(19 \%)$, sore throat (15\%). These findings are similar with the findings of other studies conducted in Bangladesh. ${ }^{9,13}$ In present study, Fever was the dominant symptom. 216 (72\%) patients presented with fever which was consistent with Guan et al, ${ }^{12}$ Wang et al, ${ }^{15}$ Zhang et al. ${ }^{16}$

In present study, $34 \%$ of patients had one or more than one co-morbidity where majority had HTN (20\%) and diabetes (18\%) followed by heart disease $(6 \%)$, renal disease $(9 \%)$, COPD (7\%), liver disease (4\%) and obesity (6\%). Similarly, in other studies conducted in Bangladesh and other countries, HTN and DM were found to be the two most common comorbidities among patients. ${ }^{9,}$ 17-19

In present study, Death rate was $2 \%$. This is consistent with the finding of other study conducted in Bangladesh observed that 1\% death in their study. ${ }^{19}$

Death is associated with the presence of a significantly higher proportion of comorbidities (any) (97 vs. 5 for alive and dead respectfully. Overall recovery from ICU was poor and higher number of deaths counted in this group.

In our study, supporting the result, we observed that male sex, presence of comorbidities, requirement of ICU support was significantly associated with mortality. Yang et al. also assessed same issue and found that overall prevalence of comorbidities in the COVID 19 patients and found that underlying disease, including HTN, respiratory system disease, cardiovascular disease could be significant risk factors for severe patients compared with non-severe patients. ${ }^{20}$

\section{Conclusion:}

In our session COVID 19 affects male more than female. Common symptoms are fever, cough, breathlessness, dysgeusia, anosmia etc. $34 \%$ of patients had comorbidity and death rate was $2 \%$. Diagnosing the patients by clinical presentation and confirmed by RT-PCR lead us to give proper treatment and reduce the morbidity and mortality rate as well as reducing the community transmission of SARS CoV-2 by isolation of COVID positive patient.

\section{Conflict of Interest - None.}

\section{References:}

1. Zhong B, Luo W, Li $\mathrm{H}$ et al. Knowledge, attitudes, and practices towards COVID-19 among Chinese residents during the rapid rise period of the COVID-19 outbreak: a quick online cross-sectional survey. Int J Biol Sci. 2020; 16(10): 1745-1752. DOI: 10.7150/ijbs.45221 
2. Ruan S. Likelihood of survival of coronavirus disease 2019. The Lancet Infectious Diseases. 2020; 20(6): 630631. DOI: 10.1016/s1473-3099(20)30257-7

3. Lu H, Stratton C, Tang Y. Outbreak of pneumonia of unknown etiology in Wuhan, China: The mystery and the miracle. J Med Virol. 2020; 92(4): 401-402. DOI: 10.1002/jmv.25678

4. Repository.unj.ac.id. http://repository.unj.ac.id/10883/7/ DAFTAR\%20PUSTAKA\%20.pdf. Published 2021. Accessed August 2, 2021.

5. Yang F, Liu N, Hu JY, et al. Zhonghua Jie He He Hu Xi Za Zhi. 2020; 43(3): 180-182. DOI: 10.3760/ cma.j.issn.1001-0939.2020.03.007

6. Chen ZM, Fu JF, Shu Q, et al. Diagnosis and treatment recommendations for pediatric respiratory infection caused by the 2019 novel coronavirus. World J Pediatr. 2020; 16(3): 240-246. DOI: 10.1007/s12519-020-00345-5

7. World Health Organization (WHO). Coronavirus disease (COVID- 2019) Bangladesh situation reports - 24 . 2020[Cited 2020August 25].1-17. Available from: http// www.who.int/docs/default-source/searo/Bangladesh/ covid-19-who-bangladesh-situation-reports/ who-covid19 update-26-2020824.pdf.

8. World Health Organization (WHO). Coronavirus disease (COVID- 2019) Pandemic 2020 [cited 2020 Aug 15] Available from: http//www.who.int/ emergencies/ diseases/ novel coronavirus-2019.

9. Mowla S, Azad K, Kabir A et al. Clinical Profile of 100 Confirmed COVID-19 Patients Admitted in Dhaka Medical College Hospital, Dhaka, Bangladesh. Journal of Bangladesh College of Physicians and Surgeons. 2020: 29-36. DOI:10.3329/jbcps. v38i0.47445.

10. Nasiri K, Dimitrova A. Comparing saliva and nasopharyngeal swab specimens in the detection of COVID-19: A systematic review and meta-analysis. $J$ Dent Sci. 2021;16(3): 799-805. DOI: 10.1016/ j.jds.2021.01.010

11. National guideline on Clinical Management of coronavirus disease 2019 (COVID 19). Disease Control Division Directorate General of Health Services and Ministry of Health and Family welfare. Government of the People's republic of Bangladesh, 2020.2020 [cited
202 Aug 26]. /c/users/USER/downloads/COVIDguideline-V4.303.2020.pdf.

12. Gupta N, Agrawal S, Ish $\mathrm{P}$ et al. Clinical and epidemiologic profile of the initial COVID-19 patients at a tertiary care centre in India. Monaldi Archives for Chest Disease. 2020;90(1). DOI:10.4081/monaldi.2020.1294

13. Hossain M, Mark S, Kabir A, Das P, Islam M, Das A. An Epidemiological Study of Laboratory Confirmed COVID19 Cases Admitted in Dhaka Medical College Hospital. $J$ Med. 2020; 21(2): 69-75. DOI: 10.3329/jom. v21i2.50208

14. IEDCR COVID - 19 status of Bangladesh. https:// www.iedcr.gov. bdindex.php/component/content/article/ 73-nCov-2019.

15. Wang D, $\mathrm{Hu} \mathrm{B}, \mathrm{Hu} \mathrm{C}$ et al. Clinical Characteristics of 138 Hospitalized Patients With 2019 Novel CoronavirusInfected Pneumonia in Wuhan, China. JAMA. 2020; 323(11): 1061. DOI: 10.1001/jama.2020.1585.

16. Zhang J, Dong X, Cao Y et al. Clinical characteristics of 140 patients infected with SARS CoV 2 in Wuhan, China. Allergy. 2020; 75(7): 1730-1741. DOI: 10.1111/ all.14238

17. Nelson L, Simard J, Oluyomi A et al. US Public Concerns About the COVID-19 Pandemic from Results of a Survey Given via social media. JAMA Intern Med. 2020; 180(7): 1020. DOI:10.1001/jamainternmed. 2020.1369

18. Singh A, Gupta R, Misra A. Comorbidities in COVID19: Outcomes in hypertensive cohort and controversies with renin angiotensin system blockers. Diabetes \& Metabolic Syndrome: Clinical Research \& Reviews. 2020; 14(4): 283-287. DOI: 10.1016/j.dsx.2020.03.016

19. Ahmed N, Islam M, Kabir M, Rahman M, Sadat S. Clinico-Pathological Findings of Bangladeshi Covid 19 Patients with their Clinical Outcome: Study of A Cohort of 201 Cases. Journal of Bangladesh College of Physicians and Surgeons. 2020:37-42. DOI:10.3329/ jbcps. v38i0.47346

20. Yang J, Zheng Y, Gou X et al. Prevalence of comorbidities and its effects in patients infected with SARS-CoV-2: a systematic review and metaanalysis. International Journal of Infectious Diseases. 2020; 94: 91-95. DOI: 10.1016/j.ijid.2020.03.017 